\title{
Diagnostic Value of Digital Breast Tomosynthesis Combined with Magnetic Resonance Imaging for Breast Cancer in Dense Breast
}

\section{Tissues}

\author{
Hongfang $\mathrm{Xu}^{1}$, Wei Zeng ${ }^{1,{ }^{*}}$, Zehong $\mathrm{Fu}^{1}$ and Qing Cui ${ }^{1}$ \\ ${ }^{1}$ Department of Radiology, Affiliated Hospital of Jianghan University, The Sixth Hospital of Wuhan, Wuhan, China \\ "Corresponding author: Department of Radiology, Affiliated Hospital of Jianghan University, The Sixth Hospital of Wuhan, Wuhan, China. Email: qggs297157@163.com
}

Received 2021 April 04; Revised 2021 September 18; Accepted 2021 September 19.

\begin{abstract}
Background: Early diagnosis and timely treatment are crucial for breast cancer patients.

Objectives: This study aimed to investigate the diagnostic value of full-field digital mammography (FFDM), digital breast tomosynthesis (DBT), and magnetic resonance imaging (MRI) for breast cancer.

Patients and Methods: This study was performed on 210 patients diagnosed with breast cancer and benign breast lesions ( $\mathrm{n}=$ 105) by FFDM, DBT, MRI, and pathological examination from January 2019 to December 2020. The patients' imaging and clinical data were retrospectively analyzed. The lesions were evaluated according to the breast imaging-reporting and data system, with pathological diagnosis as the gold standard. The diagnostic efficiency of the examination methods was analyzed by plotting the receiver operating characteristic (ROC) curves. The DBT and MRI results were finally compared.

Results: In 210 patients, 105 benign and 105 malignant lesions were detected. The area under the ROC curve (AUC) of FFDM, DBT, MRI, FFDM + DBT, and FFDM + MRI was 0.734, 0.857, 0.883, 0.865, and 0.924, respectively. Based on the results, the AUC values were significantly higher for DBT, MRI, FFDM + DBT, and FFDM + MRI compared to FFDM $(\mathrm{P}<0.05)$, while similar values were reported for the former methods $(\mathrm{P}>0.05)$. The diagnostic sensitivity of MRI was higher than that of DBT and FFDM; the sensitivity of DBT was higher than that of FFDM; and the specificity and positive predictive value were higher for DBT compared to MRI and FFDM.

Conclusion: Compared to FFDM, DBT and FFDM + DBT could significantly improve the diagnostic efficiency of breast cancer; the diagnostic efficiency of these modalities was comparable to that of MRI and FFDM + MRI. The sensitivity of DBT was lower than that of MRI and higher than that of FFDM, while its specificity and positive predictive value were higher than those of MRI. Overall, FFDM + DBT and FFDM + MRI are conducive to early diagnosis.
\end{abstract}

Keywords: Breast Cancer, Full-Field Digital Mammography, Digital Breast Tomosynthesis, Magnetic Resonance Imaging

\section{Background}

Breast cancer currently ranks the first most common female malignant cancer, posing a serious threat to women's health. Therefore, early diagnosis and timely treatment are essential for all breast cancer patients (1, 2). In recent years, imaging technologies have played an important role in the early diagnosis of breast cancer (3). Magnetic resonance imaging (MRI), as the most sensitive imaging method for breast cancer, can be used to evaluate breast lesions in terms of morphology, blood perfusion, water diffusion, and biochemical metabolism through multiple scanning sequences. However, due to low specificity, lack of microcalcification detection, long duration, and selectivity for patients, it is difficult to apply this method in most primary healthcare units.

Digital breast tomosynthesis (DBT), which can significantly improve the detection rate of breast lesions, has been applied to screen breast cancer since 2011. Breast lesions with different shapes can be clearly visualized in different positions using three-dimensional (3D), multilayered, projection images (slice thickness: $1 \mathrm{~mm}$ ) at different angles. Compared to other methods, DBT is more specific and sensitive in the detection of breast lesions, especially dense breast tissues. It is a novel imaging modality which has replaced full-field digital mammography (FFDM) in the diagnosis and screening of breast cancer in some regions (4-7). 


\section{Objectives}

In this study, the diagnostic efficiencies of FFDM, DBT, MRI, FFDM + DBT, and FFDM + MRI for breast cancer were compared to evaluate their diagnostic value.

\section{Patients and Methods}

\subsection{General Data}

The clinical data of patients, who underwent pathological examinations in our hospital from January 2019 to December 2020, were retrospectively analyzed. The inclusion criteria were as follows: (1) undergoing DBT, FFDM, and MRI before surgery or biopsy; and (2) having no history of breast surgery, radiotherapy, or chemotherapy before examinations. On the other hand, the exclusion criteria were as follows: (1) unclear images or incomplete data that could not be analyzed; and (2) a history of breast surgery, radiotherapy, or chemotherapy before examinations. All enrolled patients $(n=210)$ were women aged 26 - 80 years, with an average age of 53 years. Nipple discharge was detected in 32 cases; breast pain was reported in 83 cases; palpable mass was reported in 198 cases; skin dimpling was reported in two cases; and nipple retraction was found in two cases.

\subsection{X-ray Examination of Breast Tissue}

The patients underwent FFDM with bilateral craniocaudal (CC) and mediolateral oblique (MLO) views of the breasts and DBT with CC and MLO views of the affected breast on a GE Senographe Essential DBT X-ray machine (USA). The imaging process of DBT included nine low Xray exposures around the breasts, with an X-ray tube at a scanning angle of $25^{\circ}$ to obtain a series of low-dose, twodimensional (2D) images. Subsequently, the images were reconstructed using a computer post-processing software to obtain a series of three-dimensional tomographic images with different slice thicknesses and 2D V-Preview images.

\subsection{MRI Examination of Breast Tissue}

A GE Discovery MR750 3.0T system (USA) and fourchannel, phased-array coils were used for MRI examinations. The prone position with advanced feet was used to make the breasts symmetrical in the coils. The imaging parameters and sequences were as follows: (1) axial T2-IDEAL sequence: repetition time (TR): $6600 \mathrm{~ms}$, echo time (TE): 43 $\mathrm{ms}$, inversion time (TI): $145 \mathrm{~ms}$, field of view (FOV): $33 \times 33$ $\mathrm{cm}$, slice thickness: $5 \mathrm{~mm}$, layer spacing: $1 \mathrm{~mm}$, and matrix size: $320 \times 192$ pixels; (2) axial T1-weighted sequence: TR: $560 \mathrm{~ms}$, TE: $10 \mathrm{~ms}$, FOV: $33 \times 33 \mathrm{~cm}$, slice thickness: $5 \mathrm{~mm}$, layer spacing: $1 \mathrm{~mm}$, and matrix size: $320 \times 224$ pixels; (3) axial diffusion-weighted imaging/echo planer imaging sequence: TR: $5600 \mathrm{~ms}$, TE: $69 \mathrm{~ms}$, FOV: $33 \times 33 \mathrm{~cm}$, slice thickness: $5 \mathrm{~mm}$, layer spacing: $1 \mathrm{~mm}$, matrix size: $128 \times 130$ pixels, and b-values: 0 and $800 \mathrm{~s} / \mathrm{mm}^{2}$; and (4) axial 3D volume imaging and axial dynamic contrast-enhanced scan: TR: 4.3 ms, TE: $1.6 \mathrm{~ms}$, FOV: $32 \times 32 \mathrm{~cm}$, slice thickness: $0.7 \mathrm{~mm}$, and matrix size: $320 \times 320$ pixels (scanning once before contrast injection and repeated six times 30 seconds after the injection). The contrast agent (gadopentetate dimeglumine) was administered at a dose of $0.2 \mathrm{~mL} / \mathrm{kg}$ body weight at a flow rate of $2.5 \mathrm{~mL} / \mathrm{s}$.

\subsection{Imaging Analysis}

The FFDM, DBT and MRI images were read by two experienced senior radiologists in a double-blinded manner. The imaging data were divided into five groups (FFDM, DBT, MRI, FFDM + DBT, and FFDM + MRI) and read five times within an interval of at least two weeks. In case of disagreement, another senior radiologist contributed to the discussion to reach a consensus. Breast lesions were diagnosed and evaluated according to the classification criteria of the Breast Imaging-Reporting and Data System (BIRADS) (2013) (8). The BI-RADS-MRI 4A category or lower indicated benign or negative lesions, while the BI-RADS-MRI $4 \mathrm{~B}$ category or higher indicated malignant or positive lesions.

\subsection{Pathological Diagnosis}

The samples were fixed, embedded, and sectioned, and then, immunohistochemical staining and hematoxylin and eosin (H\&E) staining were performed. The slices were examined by an experienced senior pathologist to identify the histopathological types. A positive pathological diagnosis indicated a histologically malignant lesion, while a negative diagnosis indicated a histologically benign lesion.

\subsection{Statistical Analysis}

SPSS Version 26.0 (IBM Corporation, Armonk, NY, USA) was used for statistical analysis. Numerical data are expressed as percentage (\%), and $\chi^{2}$ test or Fisher's exact test was used to compare the sensitivity, specificity, and accuracy of the five examination methods. The receiver operating characteristic (ROC) curve was also plotted to calculate the area under the ROC curve (AUC). Significant differences were determined using independent $t$-test. $\mathrm{P}<0.05$ indicated a statistically significant difference. 


\section{Results}

\subsection{Clinical Data}

A total of 210 lesions were found in 210 patients, diag nosed by pathological examination; all of the lesions were single breast lesions. Among 105 benign lesions, 48 were found in the left breast and 57 in the right breast. On the other hand, among 105 malignant lesions, 50 were found in the left breast and 55 in the right breast (Table 1). The imaging results of one of the patients are shown in Figure 1.

\begin{tabular}{|c|c|c|}
\hline $\begin{array}{l}\text { Benign and } \\
\text { malignant } \\
\text { breast lesions }\end{array}$ & Histopathological type & $\mathbf{N}$ \\
\hline \multirow{5}{*}{ Benign lesions } & Breast fibroadenoma & 58 \\
\hline & Adenosis & 28 \\
\hline & Cystic hyperplasia of the breast & 12 \\
\hline & Mammary tuberculosis & 2 \\
\hline & $\begin{array}{l}\text { Intraductal papilloma of the } \\
\text { breast }\end{array}$ & 5 \\
\hline \multirow{5}{*}{$\begin{array}{l}\text { Malignant } \\
\text { lesions }\end{array}$} & $\begin{array}{l}\text { Invasive ductal carcinoma of the } \\
\text { breast }\end{array}$ & 83 \\
\hline & $\begin{array}{c}\text { Invasive lobular carcinoma of the } \\
\text { breast }\end{array}$ & 7 \\
\hline & $\begin{array}{c}\text { Ductal carcinoma in situ of the } \\
\text { breast }\end{array}$ & 8 \\
\hline & $\begin{array}{c}\text { Mucinous adenocarcinoma of the } \\
\text { breast }\end{array}$ & 5 \\
\hline & Premalignant breast lesions & 2 \\
\hline
\end{tabular}

\subsection{Imaging and Pathological Results of Examination Methods}

The diagnostic efficiencies of the five examination methods in this study were analyzed for breast cancer, with pathological diagnosis as the gold standard. Among 210 patients, 105 cases had positive pathological findings, while 105 cases had negative pathological findings. Based on FFDM, there were 116 cases with positive findings and 94 cases with negative findings. Based on DBT, there were 110 cases with positive findings and 100 cases with negative findings. . Moreover, based on FFDM + DBT, there were 110 cases with positive findings and 100 cases with negative findings. Also, based on MRI, there were 127 cases with positive findings and 83 cases with negative findings. According to FFDM + MRI, there were 120 cases with positive findings and 90 cases with negative findings. In terms of the density of mammary glands in 210 patients according to the American College of Radiology (ACR), four fatty type
(ACRa), 14 scattered fibroglandular (ACRb), 174 dense and uneven (ACRc), and 18 extremely dense (ACRd) lesions were detected (Table 2).

4.3. Diagnostic Efficiencies of Five Examination Methods for Breast Cancer

The specificity, sensitivity, accuracy, negative predictive value, positive predictive value, and Youden's index of the five examination methods were compared. MRI had a higher diagnostic sensitivity compared to DBT and FFDM; DBT had a higher diagnostic sensitivity compared to FFDM; and DBT had higher specificity and positive predictive value compared to MRI and FFDM. Besides, FFDM + MRI had a higher diagnostic sensitivity compared to FFDM and FFDM + DBT; FFDM + DBT had higher sensitivity compared to FFDM; and FFDM + DBT had a higher positive predictive value compared to FFDM + MRI and FFDM.

With pathological diagnosis as the gold standard, the diagnostic efficiencies of the five examination methods were analyzed by the ROC curve analysis (Figure 2). The descending order of AUCs for the five examination methods was as follows: FFDM + MRI (AUC, 0.924) > MRI (AUC, 0.883) $>$ FFDM + DBT (AUC, 0.865) > DBT (AUC, 0.857) > FFDM (AUC, 0.734). The diagnostic efficiency of DBT, MRI, FFDM + DBT, and FFDM + MRI was significantly higher than that of $\operatorname{FFDM}(\mathrm{P}<0.05)$, while the former four methods had similar AUCs $(\mathrm{P}>0.05)$.

\section{Discussion}

FFDM is currently recognized as an optimal imaging technology to detect breast tumors. However, the visibility of lesions decreases due to overlap with glandular tissues, leading to the reduction of diagnostic sensitivity and specificity (9). Traditional mammography provides 2D tomographic images that may not detect signs of high diagnostic value, such as burrs or lobes, and may yield false images of malignant lesions, inevitably increasing the false negative and false positive rates (10).

DBT, as a novel technology that combines traditional tomography with digital image processing, can represent the mammary glands from different angles. The lowdose projection data are reconstructed in a computer postprocessing software to acquire mammary gland images at any depth parallel to the detector level (11). Compared to traditional FFDM, DBT avoids the overlap of normal glandular tissues with the lesion in imaging, especially for dense mammary glands, improves the visibility of lesion 

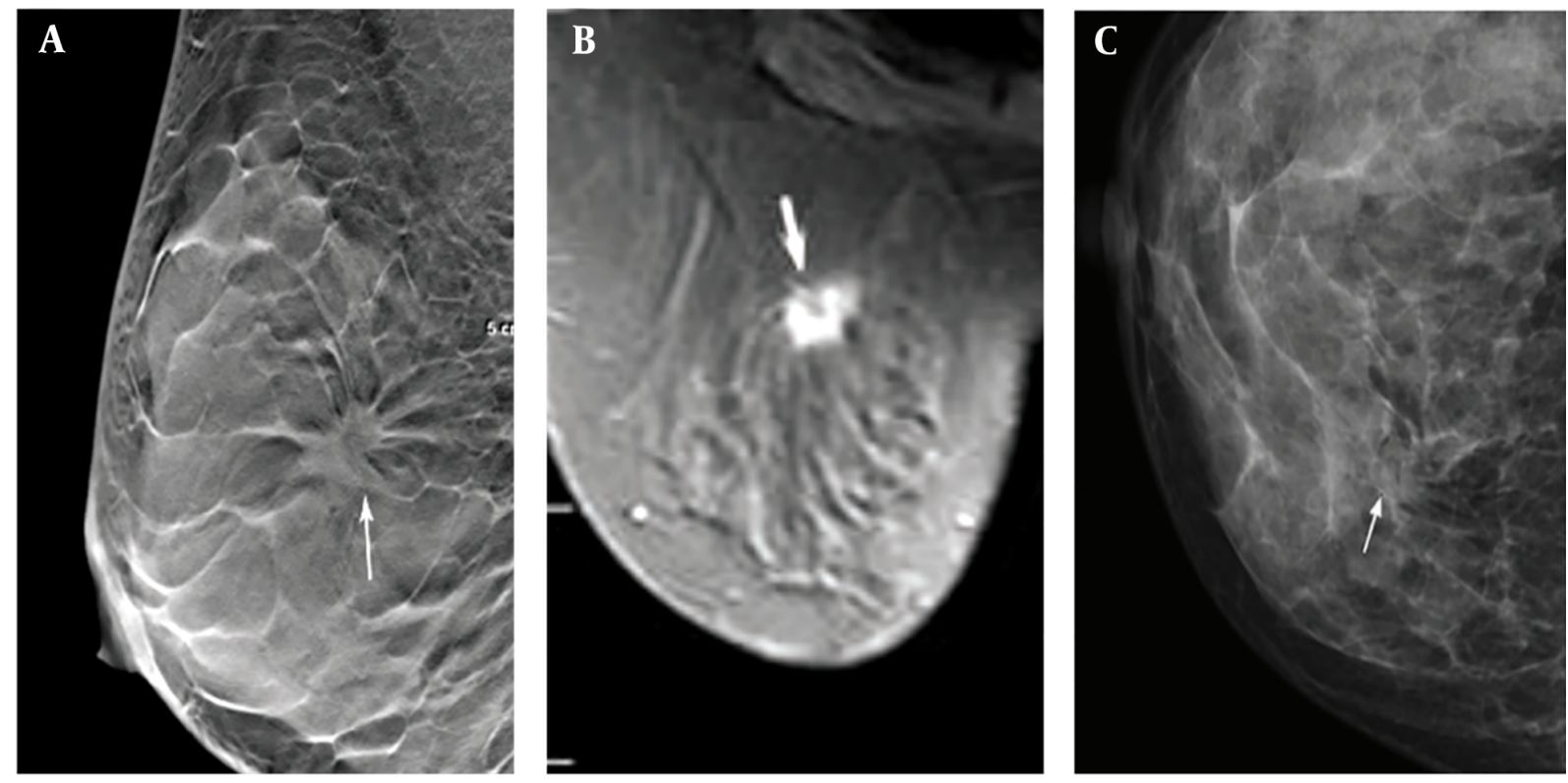

Figure 1. The imaging results of a 45-year-old female patient with a dense, uneven tumor (ACRc) diagnosed as stage II invasive ductal carcinoma. A, The DBT image of the largest layer indicates burrs on the edges of the mass (arrow). B, Fat-suppression T1-weighted MRI image (arrow). C, FFDM image showing overlaps between the mass edges and the mammary gland (arrow). ACR, American College of Radiology; DBT, digital breast tomosynthesis; FFDM, full-field digital mammography; MRI, magnetic resonance imaging.

\begin{tabular}{|c|c|c|c|c|c|c|c|}
\hline Methods & Sensitivity & Specificity & Accuracy & $\begin{array}{c}\text { Positive predictive } \\
\text { value }\end{array}$ & $\begin{array}{c}\text { Negative predictive } \\
\text { value }\end{array}$ & Youden's index & $\operatorname{AUC}(95 \% \mathrm{CI})$ \\
\hline FFDM & $82.86(87 / 105)$ & $76.19(80 / 105)$ & $78.57(165 / 210)$ & $75.00(87 / 116)$ & $82.98(78 / 94)$ & 51.83 & $0.734(0.723-0.845)$ \\
\hline DBT & $90.48(95 / 105)$ & $88.57(93 / 105)$ & $89.05(187 / 210)$ & $86.36(95 / 110)$ & $91.00(91 / 100)$ & 72.18 & $0.857(0.794-0.873)$ \\
\hline FFDM + DBT & $92.38(97 / 105)$ & $90.48(95 / 105)$ & $90.95(191 / 210)$ & $88.18(97 / 110)$ & $93.00(93 / 100)$ & 76.45 & $0.865(0.801-0.881)$ \\
\hline MRI & $100.00(105 / 105)$ & $80.95(85 / 105)$ & $91.90(193 / 210)$ & $82.68(105 / 127)$ & $100.00(83 / 83)$ & 77.94 & $0.883(0.846-0.912)$ \\
\hline FFDM + MRI & $100.00(105 / 105)$ & $86.67(91 / 105)$ & $95.24(200 / 210)$ & $87.50(105 / 120)$ & $100.00(90 / 90)$ & 83.45 & $0.924(0.876-0.986)$ \\
\hline
\end{tabular}

Abbreviations: AUC, area under curve; CI, confidence interval; DBT, digital breast tomosynthesis; FFDM: full-field digital mammography; MRI, magnetic resonance imaging.

edges (lobes or burrs), and clearly displays lesions with different shapes, heights, and positions hidden in the fibrous gland; therefore, it increases the detection rate of cancer lesions, as well as the sensitivity and accuracy of diagnosis, while reducing the false positive rate (12).

DBT can clearly display the lesion edges (burrs), suggesting its higher diagnostic accuracy and detection rate for early breast cancer, especially dense breast tissues (13), as confirmed in the present study. DBT combined with FFDM seems to have a higher diagnostic accuracy for breast cancer compared to FFDM alone (14). In the present study, the sensitivity, specificity, and accuracy of FFDM combined with DBT for the diagnosis of breast cancer were 92.38, 90.48 , and $90.95 \%$, respectively, which were higher than those reported for FFDM or DBT alone. Although DBT performs better in the detection of lesions and has a higher diagnostic accuracy for breast cancer compared to FFDM, its application is still limited due to increased radiation dose and image readings.

MRI can provide high-resolution images of soft tissues, as well as multi-parameter, multi-sequence, and multiorientation images. Dynamic contrast-enhanced MRI is recognized as the most adequate imaging technology for the examination of breast tissue (15). However, the results of the present study showed $100 \%$ sensitivity and only $80.95 \%$ specificity for MRI, probably because of the overlap in the MRI signs of benign and malignant breast lesions or insensitivity of MRI to calcification. Traditional mammog- 


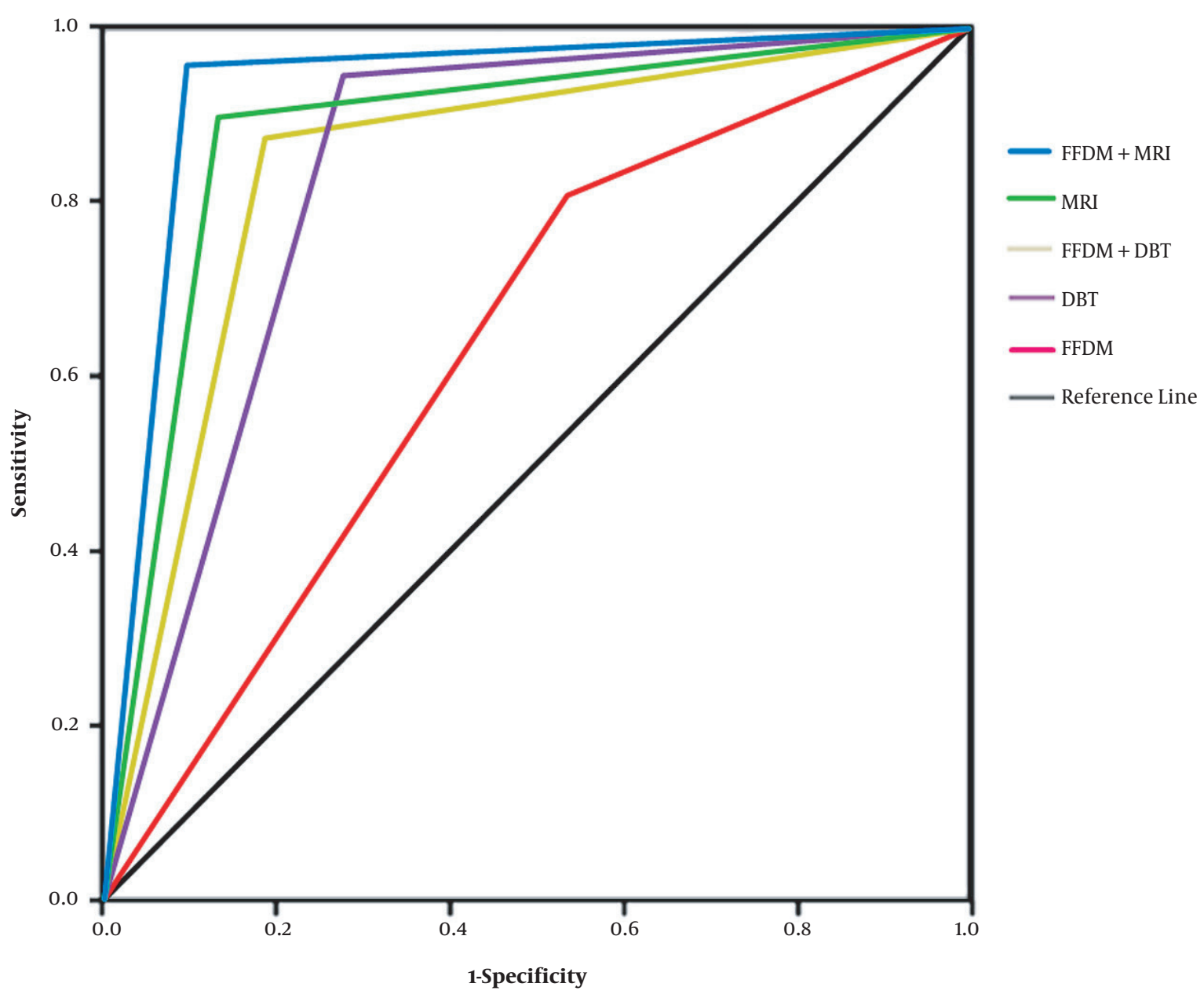

Figure 2. The results of the ROC curve analysis for the diagnostic efficiency of five examination methods for breast cancer (ROC: receiver operating characteristic curve).

raphy combined with MRI shows a higher diagnostic accuracy for breast cancer, which benefits early diagnosis (16). In the present study, the sensitivity, specificity, and accuracy of FFDM combined with MRI for breast cancer diagnosis were $100.0,86.67$, and $95.24 \%$, respectively, which were higher than those of FFDM or MRI alone. Although MRI seems to have a higher diagnostic efficacy for breast cancer, its application is still limited due to contraindications, long duration, and high cost.

Since BI-RADS category 4 is subdivided into $4 \mathrm{~A}, 4 \mathrm{~B}$, and $4 \mathrm{C}$ subcategories in the 2013 BI-RADS edition, and only 2 - $10 \%$ of $4 \mathrm{~A}$ lesions are likely to be malignant, in the current study, most lesions were considered to be benign. In this study, BI-RADS-MRI $\geq 4 \mathrm{~B}$ was defined as malignant lesions to increase pathological relevance. Among five imaging methods, FFDM + MRI had the highest diagnostic effi- ciency. FFDM + DBT and FFDM + MRI had a markedly higher diagnostic accuracy for breast cancer compared to FFDM.

This study had some limitations. First, the sample size was small $(n=210)$; therefore, the results need to be confirmed in a larger population. Second, DBT is a novel technology in China, which has not been widely applied; therefore, its advantages remain to be fully validated.

In conclusion, DBT and FFDM + DBT could significantly improve breast cancer diagnosis compared to FFDM; the former methods showed comparable diagnostic efficiencies to MRI and FFDM + MRI. The sensitivity of DBT was lower than that of MRI and higher than that of FFDM, while its specificity and positive predictive value were higher than those of MRI. All of the examination methods had certain diagnostic values, with the highest diagnostic efficiency found in FFDM + MRI. Besides, FFDM + DBT and FFDM 
+ MRI could improve the diagnostic accuracy of breast cancer, which is conducive to early diagnosis.

\section{Acknowledgments}

We would like to thank the coauthors of this study for their significant contributions to this study.

\section{Footnotes}

Authors' Contribution: Hongfang Xu and Wei Zeng designed this study and significantly revised the manuscript; and Zehong Fu and Qing Cui performed this study and drafted the manuscript.

Conflict of Interests: The authors declare no conflicts of interest.

Ethical Approval: This study was approved by the ethics committee of the Sixth Hospital of Wuhan, Affiliated Hospital of Jianghan University, Wuhan, China.

Funding/Support: This study was not financially supported.

Informed Consent: Written informed consent was obtained from all participants.

\section{References}

1. Qaseem A, Lin JS, Mustafa RA, Horwitch CA, Wilt T]; Clinical Guidelines Committee of the American College of Physicians, et al. Screening for Breast Cancer in Average-Risk Women: A Guidance Statement From the American College of Physicians. Ann Intern Med. 2019;170(8):54760. doi: 10.7326/M18-2147. [PubMed: 30959525].

2. Wang L. Early Diagnosis of Breast Cancer. Sensors (Basel). 2017;17(7). doi: 10.3390/s17071572. [PubMed: 28678153]. [PubMed Central: PMC5539491].

3. Alzaghal AA, DiPiro PJ. Applications of Advanced Breast Imaging Modalities. Curr Oncol Rep. 2018;20(7):57. doi: 10.1007/s11912-018-07003. [PubMed: 29845403].

4. Cott Chubiz JE, Lee JM, Gilmore ME, Kong CY, Lowry KP, Halpern EF, et al. Cost-effectiveness of alternating magnetic resonance imaging and digital mammography screening in BRCA1 and BRCA2 gene mutation carriers. Cancer. 2013;119(6):1266-76. doi: 10.1002/cncr.27864. [PubMed: 23184400]. [PubMed Central: PMC3586945].
5. Svahn TM, Houssami N, Sechopoulos I, Mattsson S. Review of radiation dose estimates in digital breast tomosynthesis relative to those in two-view full-field digital mammography. Breast. 2015;24(2):93-9. doi: 10.1016/j.breast.2014.12.002. [PubMed: 25554018]. [PubMed Central: PMC5064843].

6. Rafferty EA, Durand MA, Conant EF, Copit DS, Friedewald SM, Plecha DM, et al. Breast Cancer Screening Using Tomosynthesis and Digital Mammography in Dense and Nondense Breasts. JAMA. 2016;315(16):1784-6. doi: 10.1001/jama.2016.1708. [PubMed: 27115381].

7. Lee WK, Chung J, Cha ES, Lee JE, Kim JH. Digital breast tomosynthesis and breast ultrasound: Additional roles in dense breasts with category 0 at conventional digital mammography. Eur $J R a$ diol. 2016;85(1):291-6. doi: 10.1016/j.ejrad.2015.09.026. [PubMed: 26499000].

8. Mercado CL. BI-RADS update. Radiol Clin North Am. 2014;52(3):481-7. doi: 10.1016/j.rcl.2014.02.008. [PubMed: 24792650].

9. Mori M, Akashi-Tanaka S, Suzuki S, Daniels MI, Watanabe C, Hirose M, et al. Diagnostic accuracy of contrast-enhanced spectral mammog raphy in comparison to conventional full-field digital mammography in a population of women with dense breasts. Breast Cancer 2017;24(1):104-10. doi: 10.1007/s12282-016-0681-8. [PubMed: 26942415].

10. Sechopoulos I. A review of breast tomosynthesis. Part I. The image acquisition process. Med Phys. 2013;40(1):14301. doi: 10.1118/1.4770279. [PubMed: 23298126]. [PubMed Central: PMC3548887].

11. Sankatsing VDV, Juraniec K, Grimm SE, Joore MA, Pijnappel RM, de Koning HJ, et al. Cost-effectiveness of Digital Breast Tomosynthesis in Population-based Breast Cancer Screening: A Probabilistic Sensitivity Analysis. Radiology. 2020;297(1):40-8. doi: 10.1148/radiol.2020192505. [PubMed: 32749212]. [PubMed Central: PMC7526946].

12. Michell MJ, Batohi B. Role of tomosynthesis in breast imaging going forward. Clin Radiol. 2018;73(4):358-71. doi: 10.1016/j.crad.2018.01.001. [PubMed: 29415806]

13. Krammer J, Zolotarev S, Hillman I, Karalis K, Stsepankou D, Vengrinovich V, et al. Evaluation of a new image reconstruction method for digital breast tomosynthesis: effects on the visibility of breast lesions and breast density. Br J Radiol. 2019;92(1103):20190345 doi: 10.1259/bjr.20190345. [PubMed: 31453718]. [PubMed Central PMC6849672].

14. Seo M, Chang JM, Kim SA, Kim WH, Lim JH, Lee SH, et al. Addition of Digital Breast Tomosynthesis to Full-Field Digital Mammography in the Diagnostic Setting: Additional Value and Cancer Detectability. J Breast Cancer. 2016;19(4):438-46. doi: 10.4048/jbc.2016.19.4.438. [PubMed: 28053633]. [PubMed Central: PMC5204051].

15. Jochelson MS, Dershaw DD, Sung JS, Heerdt AS, Thornton C, Moskowitz $\mathrm{CS}$, et al. Bilateral contrast-enhanced dual-energy digital mammog raphy: Feasibility and comparison with conventional digital mammography and MR imaging in women with known breast carcinoma. Radiology. 2013;266(3):743-51. doi: 10.1148/radiol.12121084. [PubMed: 23220903]. [PubMed Central: PMC5673037].

16. Xiang W, Rao H, Zhou L. A meta-analysis of contrast-enhanced spectral mammography versus MRI in the diagnosis of breast cancer. Thorac Cancer. 2020;11(6):1423-32. doi: 10.1111/1759-7714.13400. [PubMed: 32233072]. [PubMed Central: PMC7262891]. 